\title{
Avaliação do custo do tratamento de úlceras por pressão em pacientes hospitalizados usando curativos industrializados
}

\author{
Evaluation of the cost of treating pressure ulcers \\ in hospitalized patients using industrialized dressings
}

Angela Cristina Beck Lima ${ }^{1}$

Diana M endonça Guerra²

${ }^{1}$ Laboratório Farmacêutico do Estado de Pernambuco. Largo Dois I rmãos 1.117, DoisIrmãos. 52171-010 Recife PE.

beck.angela@hotmail.com ${ }^{2}$ Programa de Residência em Planejamento e Gestão deServiços Farmacêuticos, Secretaria de Saúde do Estado de Pernambuco.
Abstract This work evaluated wound dresses used in the N eurosurgery D epartment of Restauração H ospital: polyurethane, hydrogel and activated carbon wound dresses and hydrogel with alginate used for pressure ulcer care. This work aimed to identify a critical factor that increases demand and cost of wound dresses. The evaluation conducted at the Neurosurgery Department identified individuals at risk of pressureulcer development. Sixty-two patients wereevaluated and the prevalence of pressure ulcer was $22.6 \%$ according to the Braden scale. Comparative evaluation between patients that didn't receive preventive measures and others that received, showed that the average daily cost of hospitalization for the first group was $45 \%$ higher than the mean for the second group. The Wilcoxon-M ann-Withiney test compared the population at risk to develop pressure ulcer and population at low risk showing that the evaluation of Braden Scale scores between the groups presents statistically significant differences and confidencelimits of $95 \%$. Pressure ulcer is a key quality indicator in health services. It is possibleto reduce costs and offer higher quality public health services by implementing a training program of nursing staff using a preventive measure protocol based on a test to evaluate risk as Braden Scale.

Key words Pressure ulcer, Prevention, Braden Scale, Wound dress, Prevalence
Resumo Este trabalho avaliou o uso de curativos industrializados: poliuretano, hidrogel, carvão ativo e também hidrogel com alginato, no tratamento de úlcera por pressão na Clínica N eurocirúrgica do H ospital da Restauração. 0 objetivo foi identificar o fator crítico queaumenta a demanda ecustos com curativos industrializados. A avaliação, realizada na Clínica N eurocirúrgica, identificou os indivíduos que apresentaram risco de desenvolver úlcera por pressão. 62 pacientes foram avaliadosea prevalência foi de $22,6 \%$, segundo escore obtido na escala de Braden. A avaliação comparativa entre indivíduos que receberam medidas preventivas e entre os que não receberam mostrou queo custo médio diário dehospitalização para o primeiro grupo foi $45 \%$ maior que para o segundo grupo. 0 teste de Wilcoxon-M ann-Withiney comparou a população derisco com a população debaixo risco, mostrando quea análisedosescores da Escala deBraden entreos grupos apresenta diferenças estatisticamente significativas quando estas duas populações são comparadas, intervalo deconfiança de $95 \%$. Ú Icera por pressão é um indicador de qualidade dos serviços de saúde. Pode-se reduzir custo e oferecer serviços públicosdemaior qualidadeseforem implantados treinamentos com a equipedeenfermagem, usando um protocolo de medidas preventivas baseado em um teste deavaliação de risco como a escala de Braden. Palavras-chaveÚ Icera por pressão, Prevenção, Escala de Braden, Curativos industrializados, Prevalência. 


\section{Introdução}

O uso dos fundamentos na área econômica a fim de projetar o uso racional de materiais médico-hospitalares é de fundamental importância à administração dos recursos das instituições públicas de saúde no Brasil, tendo em vista que as despesas nesta área são crescentes, enquanto os recursos disponibilizados não aumentam na mesma proporção.

A partir dos anos 80 , surge no Brasil o conceito do cidadão como cliente. A ideia central consisteem voltar as organizações públicas para seus clientes, tornando-as preocupadas com a acessibilidadee aqualidade dos serviços prestados. Esta é a administração com o foco no cidadão, e alguns de seus objetivos são aumentar a qualidade dos serviços visando diminuir desperdícios, aumentar a satisfação dos cidadãos com os serviços prestados e racionalizar custos através do incremento de performance'.

A política de saúde no Brasil é do tipo assistencialista, eo direito à saúdeédefinido na Constituição Federal: "dever do Estado e direito do cidadão". No entanto, essa política tem se tornado bastante onerosa ao Estado devido à grande demanda criada pelo crescimento populacional, mas também devido a vários problemas administrativos e desperdícios.

A incidência deúlcera por pressão éconside rada como um indicativo da qualidade dos cuidados. Sua presença é associada ao aumento do tempo de internamento, de carga de trabal ho para enfermagem e aumento de custos, além de maior morbidade e mortalidade aos pacientes internados².

É necessário que se investigue o nível de risco de indivíduos em adquirir úlcera por pressão, bem como a prevalência dela na clínica e os custos atuais com curativos de modo a melhorar os serviço de saúde e minimizar os custos com curativos industrializados.

Em 1962, Norton e colaboradores desenvolveram uma escala que abrange cinco parâmetros derisco para desenvolvimento deúlcera por pressão (UP): condições físicas, condições mentais, atividade, mobilidadeeincontinência, eGosnell ${ }^{3}$, em 1973, revisa e modifica a Escala de Norton e utiliza quatro de suas cinco subescal as e adiciona itens relacionados à avaliação da integridade da pele, isto é: aparência, tonalidadeesensibilidade. Além disso, a autora inclui todas as medicações tomadas pelo paciente.

Waterlow4, em 1985, desenvolveu um guia para avaliação de pacientes com risco de desen- volver UP, contendo também condutas preventivas e terapêuticas. Esta escala émais utilizada no Reino Unido e classifica os pacientes como em risco, alto risco erisco muito alto.

$\mathrm{M}$ arcer ${ }^{5}$ estimou que seriam poupados imediatamente cerca de 25 milhões de libras no Reino Unido se os pacientes fossem avaliados logo na admissão e ainda fossem adotadas medidas modernas de prevenção.

A Escala de Braden foi desenvolvida por Braden e Bergstron ${ }^{6}$ como estratégia para diminuir a incidência deU P no serviço ondetrabal havam. É composta de seis subescalas: percepção sensorial, umidade da pele, atividade, mobilidade, estado nutricional, friç̧ão e cisal hamento.

Segundo Allman? ${ }^{7}$ a incidência de UP em pacienteshospitalizadosnos EUA varia de $2 \%$ a $40 \%$.

Deacordo com estudo realizado nos Estados Unidos por The National Pressure Ulcer Advisory Panel ${ }^{8}$, o custo estimado do tratamento para úlcera de pressão édeU $\$ 2.000$ a US $\$ 30.000$ por paciente, sendo o custo anual estimado em US\$ 8,5 bilhões.

Hanson et al. ${ }^{9}$ afirmaram que 1,7 milhão de pessoas desenvolvem UP anualmente nos Estados Unidos e que os custos com tratamento chegam a US\$ 8,5 bilhões ao ano. Realizaram auditoria em pacientes internados com relação ao desenvolvimento de UP após o implemento do uso de protocolos para tratamento e prevenção e verificaram que a prevalência, em 18 meses de pesquisa, foi de $14,5 \%$. De acordo com seus dados anteriores, os autores concluíram que não houve decréscimo na taxa de prevalência, talvez porque não houvesse aderência da equipe de enfermagem e da família do paciente ou porque 0 protocolo não foi efetivo. E ressaltaram a necessidade do comprometimento do treinamento periódico da equipe de enfermagem.

Knox ${ }^{10}$ demonstrou que a mudança de decúbito deve ser feita a cada duas horas. Os pacientes devem ser mudados de decúbito tomando o cuidado de não colocá-los sobre áreas hiperemiadas.

Perneger et al. ${ }^{11}$, da Universidade de Gênova, realizaram uma pesquisa em pacientes internados usando a Escala de Norton para avaliar os riscos de desenvolvimento de U P em 2.373 pacientes emedir sua incidência. Chegaram ao resultado de 277 novas UP (5,7 por mil pessoas ao dia). Concluíram quea idade do paciente eainda os fatores medidos pela Escala de N orton (condição física, condição mental, atividade, mobilidade e incontinência) foram os maiores riscos para o desenvolvimento da úlcera. 
Ooi et al..$^{12}$ determinaram como os cuidados em domicílio podem afetar o aparecimento de UP. Foram selecionados 5.518 pacientes, com idadeacima desessenta anos, de setenta instituições do programa National $\mathrm{H}$ ealth Care, que foram classificados como alto risco ou baixo risco baseado na incidência de UP e comportamento degenerativo. A incidência total com relação à UP foi de $11,4 \%$, e o risco foi 4,3 vezes maior em pacientes dealto risco queem de baixo risco. Em relação ao comportamento degenerativo, a incidência foi de $27 \%$ eo risco foi 7,1 vezes mai or no grupo de alto risco. Foram considerados neste estudo fatores socioculturais, uso de medicamentos como antipsicóticos, doenças como a diabetes, higiene, incontinência urinária, deambulação, alimentação e grau de desorientação.

Van M arum et al. ${ }^{13}$ fizeram um estudo comparativo para verificar qual escala seria mais adequada para pacientes tratados em domicílio. Foram avaliadas neste estudo tanto a Escala de Norton como a de Dutch, e eles concluíram que a Escala de Norton era mais adequada por ser simplificada e de fácil aplicação.

Courtenay et al. ${ }^{14}$ usaram larvaterapia para tratar úlceras de pacientes hospitalizados. As larvas foram deixadas na ferida por três dias, eapós o tratamento foram observadas diminuições do tamanho da ferida em $5 \%$, do tecido necrótico em $68 \%$ e aumento de tecido de granulação de até $26 \%$. Houve também uma diminuição do número de feridas com exsudato, em $33 \%$; os odores diminuíram em $81 \%$ e a dor foi reduzida em $69 \%$ dos pacientes.

0 Royal College of Nursing ${ }^{15}$ publicou em 2001 um guia para avaliar os riscos e promover a preven ção deUP, Clinical practiceguidelines. Este guia contém recomendações para estabelecer fatores de risco, identificar indivíduos em risco eos cuidados para evitar a formação de UP, bem como seu tratamento. As equipes de enfermagem devem ser treinadas para que sejam capazes de avaliar os riscos e fazer a prevenção. Devem ser criados programas interdisciplinares de educação e treinamento para o pessoal de enfermagem. Até mesmo o paciente e familiares devem ser educados para lidar com o problema. Segundo esse guia, a filosofia do tratamento está centrada no paciente e em suas necessidades individuais, bem como no trabalho de equipe.

Gómez e Rosillo ${ }^{16}$ desenvolveram uma nova escal a para avaliação de risco de desenvolvimento de UP para pacientes críticos de UTI. A nova escala inclui fatores como estado hemodinâmico, estado respiratório, consciência e mobilida- de. As autoras descartam fatores nutricionais e constitucionais por considerá-los subjetivos.

Bueno ${ }^{17}$ realizou um estudo usando ácidos graxos essenciais na prevenção de UP. O estudo foi realizado com dois grupos: um grupo experimental, no qual foi usado o ácido graxo, além das medidas preventivas habituais: higiene, limpeza da pele, mudanças de decúbito e aplicação de material redutor da pressão; e no grupo controle, foram mantidas apenas as medidas preventivas. Cada grupo continha 96 indivíduos. 0 autor relata quea incidência deUP no grupo controlefoi de $35 \%$, enquanto no grupo experimental foi de $19 \%$.

Uhlemannn et al. ${ }^{18}$ descrevem numerosos estudos realizados no tratamento de úlceras, principalmente na perna, usando ultrassom. Nesses estudos são descritos benefícios como proliferação de componentes, como fibroblastos, osteoblastos e monócitos, que agem como mediadores para a recuperação do tecido lesado. 0 ultrassom também é usado no debridamento de ferida. No entanto, os autores concluem queapesar de numerosos estudos terem sido desenvolvidose apresentarem resultados interessantes, são pouco convincentes, pois estes devem ser feitos usando uma população maior de indivíduos e ainda devem ser comparados com outros métodos. São necessários estudos mais aprofundados para que sejam definidas a intensidade ótima, o tempo de aplicação, a frequência e a duração do tratamento.

Bueno ${ }^{2}$ usou a Escala de Waterlow para avaliar os riscos de desenvolvimento deU $\mathrm{P}$ no Hospital Universitário de Pamplona, em uma amostra de 91 indivíduos cujo internamento foi superior a três dias. Como resultado, obteve $91 \%$ de pacientes com risco de desenvolver úlcera com diversos níveis de risco. A pesar do alto risco, a incidência foi baixa $(0,447 \%)$, e o autor concluiu que a baixa incidência se deve ao cumprimento das medidas preventivas.

Vários termos têm sido usados para definir úlcera por pressão: escara, úlcera de decúbito e ferida de pressão. No entanto, o termo úlcera por pressão se mostra mais adequado na medida em que se verifica ser a pressão exercida sobre os tecidos e proeminências ósseas em relação ao leito o fator etiológico mais importante nessas lesões. Desse modo, UP é definida como uma área localizada de morte celular devida à pressão por longos períodos do tecido mole, onde há uma proeminência óssea e uma superfície dura.

O metabolismo das células é dependente da circulação sanguínea para carrear os nutrientes 
necessários e remover produtos do metabolismo das células; quando não há o aporte nutricional adequado, ocorre morte celular, sendo, portanto, a pressão o principal fator de risco, apesar de existirem outros.

Outros motivos relacionados à formação de UP são: nível de consciência do paciente; insensibilidade à dor e ao desconforto causados por doença neurológica; doença vascular; incontinência urinária; má nutrição.

As UP são classificadas pelo NUAP como:

- Estágio I: Eritema não esbranquiçado de pele intacta, precursor da ulceração da pele.

- Estágio II : Lesão parcial da pele, envolvendo epiderme e/ou derme, ou ambas. A úlcera é superficial e clinicamente aparece como abrasão, bolha ou cratera rasa.

. Estágio III: Lesão total da pele, envolvendo dano ou necrose da camada subcutânea, mas não completa. A úlcera apresenta-se clinicamente como uma cratera profunda com ou sem comprometimento dos tecidos adjacentes.

. Estágio IV: Grande destruição com presença de tecidos necróticos ou dano de músculos, ossos ou estruturas de suporte, tendões e cápsula articular ${ }^{19}$.

Para diminuir a incidência deúlcera por pressão, se faz necessário um protocolo de prevenção e diminuição de riscos. Portanto, o paciente deve ser avaliado como um todo em vários aspectos, inclusive doenças preexistentes eseu estado nutricional.

A mudança de decúbito a cada duas horas, 0 tipo deleito usado para pacientes inconscientese sua higiene pessoal, bem como cuidados da incontinência urinária, são fatores decisivos para evitar úlceras por pressão.

A redução da incidência diminuiria os custos com curativos, bem como o uso de antibióticos; as equipes de enfermagem estariam comprometidas com outros cuidados que não relacionados com cuidados de feridas e ainda haveria uma grandemel hora da qualidade devida do paciente, tendo em vista que os custos causados ao paciente são intangíveis, pois não se podem mensurar os danos psicológicos eo desgaste maior que este terá pelo aumento do tempo de internamento.

\section{M etodologia}

Trata-se de um estudo de natureza observacional/descritivo/longitudinal, realizado na clínica neurocirúrgica do H ospital da Restauração. Este estudo foi submetido e aprovado pelo Comitê
deÉtica e Pesquisa enão apresentou risco para o paciente nem para os profissionais envolvidos.

A população foi composta por pacientes internados na clínica neurocirúrgica, maiores de 18 anos, com tempo de internação acima de sete dias, que apresentaram risco de desenvolver UP de acordo com a Escala de Braden.

\section{Procedimento para coleta de dados}

Levantamento de custo

por clínicas do hospital

Foi realizado um estudo retrospectivo de custo de curativos industrializados e padronizados no H ospital da Restauração, os quais foram utilizados nas diversas clínicas deste hospital com relação ao ano de 2005.

Determinação do grau de risco para UP

A pesquisana Clínica N eurocirúrgica foi realizada durante três meses e foram entrevistados 62 pacientes. Foi utilizado um formulário contendo a Escala de Braden eum questionário contendo os seguintes itens: os motivos da internação, os medicamentos utilizados, se foram realizadas mudanças de decúbito ou massagem como medidas preventivas, além do tipo de curativo utilizado indicando o início e o fim do tratamento. Essequestionário foi aplicado a cada paciente e usado para coletar dados de prontuário diariamente. Tais ferramentas foram utilizadas para acompanhar os pacientes que eram admitidos na neurocirurgia, para verificar seu risco de desenvolver UP. Depois foram incluídos os pacientes que já estavam internados devido à necessidade de verificar qual era a prevalência deUP na clínica. Todos os 62 pacientes foram avaliados de acordo com essa escala.

A Escala de Braden é um método validado para que a equipe de saúde possa avaliar os pacientes quanto ao risco de desenvolver UP, ea partir de então usar medidas preventivas. Possui escores que variam de 6 a 23 e é subdividida em subitens. Esses escores avaliam os seguintes pontos: percepção sensorial, umidade, mobilidade, nutrição efricção e cisal hamento da pele do paciente com o leito. Cada subitem da escala possui escore de 1 a 3 ou 4; quanto menor o escore, maior o risco de desenvolver UP.

O subitem percepção sen sorial é classificado como total mente limitado: escore 1 ; muito limitado: 2; levementelimitado: 3; esem limitação: 4. 
O subitem umidadeé classificado como completamente molhada: escore 1; muito molhada: 2 ; ocasionalmente molhada: 3; e raramente molhada: 4.0 subitem mobilidade é classificado como totalmenteimóvel: escore 1; bastantelimitado: 2; levemente limitado: 3; e sem limitações: 4.0 subitem nutrição muito pobre: 1 ; inadequada: 2 ; adequada: 3 ; e excelente: 4 . 0 subitem friç̧ão e cisal hamento é classificado como: problema: 1 ; problema em potencial: 2; e sem problema: 3.

$\mathrm{Nem}$ todos os pacientes internados nessa clínica apresentavam alto risco para desenvolver UP, pois mostraram escores em torno de 23, que é a pontuação máxima dessa escala.

Os pacientes de risco foram os de trauma com al gum nível deimobilidadee queapresentavam escores menores que 16, ponto crítico da escala. Foram avaliados quanto ao grau de risco os pacientes da neurocirurgia que tinham idade acima de 18 anos e ficaram internados por mais de uma semana.

Como a neurocirurgia é uma clínica que recebe pacientes de trauma e ainda pacientes com outras comorbidades, as duas populações foram comparadas para determinar se há diferenças estatísticas com relação ao grau de risco medido através da Escala de Braden, usando o teste Wilcoxon-M ann-Withiney.

Dentre os pacientes que apresentaram risco de desenvolver UP, verificaram-se quantos foram admitidos com UP e qual o grau de risco apresentado por eles.

\section{Custo do tratamento \\ paciente/dia de internamento}

0 custo do tratamento por paciente por dia de internamento foi calculado com relação a todos os medicamentos e materiais médico-hospitalares usados durante os procedimentos de cuidados da UP, bem como com relação à diária hospitalar e salários pagos às equipes médica e de enfermagem. Utilizou-se o programa Excel para computar os materiais gastos nos curativos, bem como para avaliar o custo diário do tratamento. Cada paciente acompanhado teve a úlcera por pressão acompanhada por fotografias para avaliar a evolução do quadro clínico e associá-lo ao fator de risco medido pela Escala deBraden; a classificação foi feita deacordo com 0 grau de dano tecidual. As fotografias foram utilizadas apenas para controle interno e não constam deste trabalho. Além das fotografias, foi utilizado um questionário contendo os seguintes itens: o motivo da internação, os medica- mentos utilizados, se foram realizadas mudanças de decúbito ou massagem como medida preventiva, o tipo decurativo utilizado, indicando 0 início e o fim do tratamento com o objetivo de avaliar a evolução do quadro clínico do paciente diariamente.

\section{Avaliação dos resultados}

Foi realizado o teste de M ann-Withiney para avaliar os resultados do estudo de risco, para o intervalo de confiança de $95 \%$.

\section{Resultados ediscussão}

Levantamento do custo de curativos industrializados utilizados nas diversas clínicas do Hospital da Restauração

Segundo levantamento realizado para 0 ano de 2005 do uso de curativos no Hospital da Restauração, relativo aos meses de abril a dezembro, $68 \%$ dos curativos usados na Clínica Neurocirúrgica tinham indicação para úlcera por pressão, somando mais de R\$ 25 mil (Figura 1). 0 consumo total de curativos desta clínica no mesmo ano foi de pouco mais de $\mathrm{R} \$ 26$ mil. Isto significa que o maior consumo está real mente relacionado à úlcera por pressão. 0 custo de curativos usados em UP previsto para os $12 \mathrm{me}$ ses relativos ao ano de 2005 chegaria a $\mathrm{R} \$ 33 \mathrm{mil}$.

O levantamento de custos de curativos industrializados nas diversas clínicas do Hospital da Restauração forneceu o perfil do consumo desses curativos para o hospital. A partir desses dados verificou-seque o maior consumidor desses materiais médico-hospitalares é a Clínica N eurocirúrgica. Esta parte do estudo mostra 0 custo isolado de curativos industrializados para a Clínica N eurocirúrgica, mas não demonstra o custo total do cuidado de úlceras por pressão, que inclui outros materiais médico-hospitalares e medicamentos.

\section{Classificação quanto ao risco}

de desenvolver úlcera por pressão

0 resultado da avaliação do risco de desenvolver UP usando a Escala de Braden pode ser visto na Tabela 1. Entre os pacientes de risco, que apresentaram escores menores que 16 (ponto crítico) da escala, seis pacientes (38\%) desenvolveram múltiplas úlceras em diversas áreas do corpo, com úlcera sacra de grau III. No entanto, 
sete pacientes, apesar de apresentarem os mesmos escores, haviam desenvolvido UP apenas na região sacra. Isto pode ser devido a outros fatores como a condição nutricional efísica do próprio paciente. Dos dois pacientes que apresentaram escores entre 13 e 16, um apresentou escore igual a 16, com UP grau II na região sacra; 0 outro apresentou escore igual a 13 e não desenvolveu UP. N estecaso, foi feita mudança de decúbito em horários regulares e conscientização do próprio paciente, que apresentou boa condição física enutricional. 0 estado emocional do pacientetambém contribui decisivamente para a pre venção da úlcera por pressão.

Para os pacientes de risco, o escore médio na Escala de Braden foi de 10,5 ( $D P=2,6$ ), e para a população de baixo risco o escore médio foi de $21,78(\mathrm{DP}=1,96)$.

0 teste Wilcoxon-M ann-W ithiney realizado para verificar diferenças estatisticamente significantes entre os valores centrais das duas amostras para os escores da Escala de Braden apresentou um valor de $z$ calculado de 8,8 para o intervalo de confiança de $95 \%$, enquanto o valor de $z$ tabelado $(0,05)=1,96$. Sendo o valor de $z$ calculado superior ao valor de $z$ tabelado, conclui-se que os valores médios abaixo de 16 (ponto crítico da Escala de Braden) realmente são determinantes para o desenvolvimento das úlceras por pressão, verificadas na população considerada de risco.

D os 62 pacientes entrevistados, 17 foram admitidos com UP na região sacra, ou seja, 27,4\% dos casos estudados. M as em relação à população de risco, foram 17 de 18 pacientes: 94\% foram admitidos com UP.

$\mathrm{Na}$ Tabela 2, são apresentadas as localizações das úlceras por área do corpo. Todos os pacientes que desenvolveram UP nessas áreas durante esta pesquisa foram admitidos na Neurocirurgia com UP na região sacra. N ota-sequeas maiores porcentagens se dão no maléolo e no calcanho; isto se deve ao posicionamento do paciente no leito por muitas horas em decúbito dorsal ou lateral sem mudanças periódicas. Somado a isto, $27 \%$ dos pacientes desenvolveram entre cinco e seis úlceras na região sacra, calcanho, ísquio, maléolo, occipício e joelho. 93\% dos pacientes

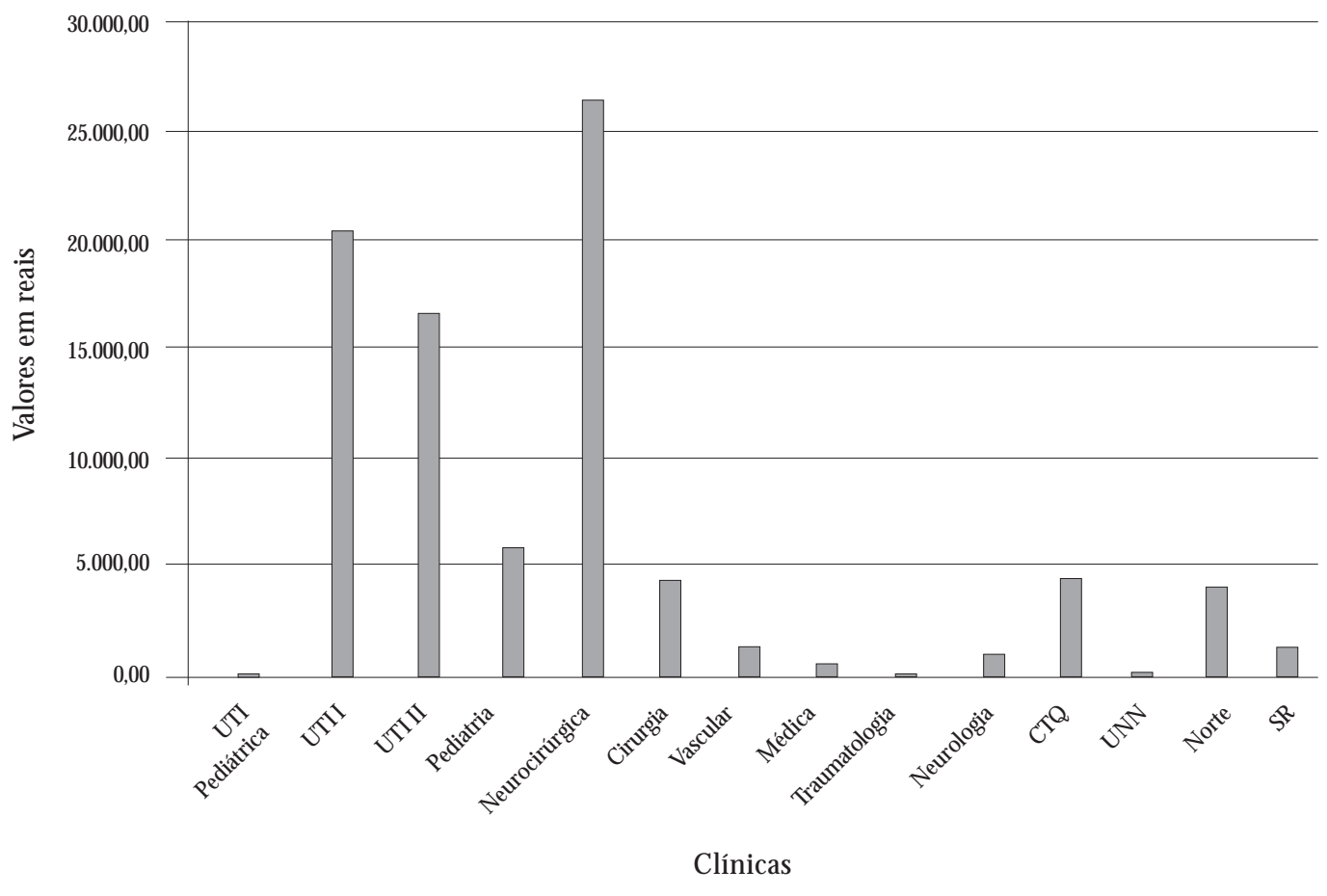

Figura 1. Custo em reais dos curativos consumidos no ano de 2005. 
que desenvolveram UP apresentaram escores na Escala de Braden iguais ou menores que 16, ponto crítico da escala.

Ainda na Tabela 2 pode ser visto que a prevalência entreos pacientes quecompõem a população de risco é de $94 \%$. A área do corpo onde ocorre a primeira injúria de tecido éa região sacra.

Tendo visto que esses pacientes em geral de senvolvem a úlcera sacra no setor de traumatologia, é bastante claro observar que a própria dificuldadedemobilidade do paciente de trauma (o paciente fica imobilizado com colar cervical em decúbito dorsal aguardando exames), associada a todos os outros fatores, como incontinência urinária, proporciona esse quadro. Um dos pacientes admitidos na Clínica N eurocirúrgica, originário da traumatologia com U P grau I, e evoluiu para grau III em apenas uma semana.

Nessa clínicafoi encontrado um tipo de pacientepadrão para desenvolver UP: paraplégico ou tetraplégico que, apesar de manter o nível normal deconsciência, não apresenta mobilidade ou sensibilidade principalmente na região pélvica e membros inferiores ou até em todo o corpo.

Cem por cento dos pacientes que desenvolveram UP apresentaram escore na Escala de Bra- den menor que 16 , sendo que $94 \%$ dos pacientes possuíam escore igual a 1 para 0 item atividade; $75 \%$ apresentaram escore para mobilidade, fricção e cisal hamento também iguais a 1 , demonstrando que estes itens da escala são preponderantes para o desenvolvimento da UP - o que está de acordo com a literatura ${ }^{20}$.

Dezessete dos 18 pacientes de risco (94\%) apresentaram UP na região sacra. A literatura cita $87,2 \%{ }^{20}$.

$\mathrm{Na}$ maioria dos casos, foi verificado que as indicações dos tratamentos por curativos eram adequadas e que em quase todos os casos elas eram seguidas adequadamente de acordo com a literatura ${ }^{19}$, exceto quando houve falta dos produtos no hospital. Ou seja, há por parte dos profissionais de enfermagem um preparo satisfatório com relação ao tratamento da úlcera já formada, mas falta ainda o preparo fundamental no sentido da prevenção da úlcera. Falta a conscientização de queaúlcera podee deveser evitada por todos os motivos óbvios discutidos anteriormente neste trabalho. Este trabalho consegue demonstrar facilmente que 0 uso de instrumentos de avaliação de risco como a Escala de Braden na avaliação do pacientelogo em sua admissão é um valioso instrumento de prevenção.

Tabela 1. Resultado da avaliação do risco de desenvolver UP usando a Escala de Braden.

\begin{tabular}{lccrc}
\hline & $\begin{array}{r}\text { Escore médio na } \\
\text { Escala de Braden }\end{array}$ & $\begin{array}{c}\text { Número de } \\
\text { pacientes }\end{array}$ & $\begin{array}{r}\text { Desvio } \\
\text { padrão }\end{array}$ & $p^{*}$ \\
\hline Pacientes com baixo risco de desenvolver UP & 21,78 & 47 & 1,96 & 0,025 \\
Pacientes com alto risco de desenvolver UP & 10,5 & 18 & 2,6 & \\
\hline
\end{tabular}

${ }^{*}$ TesteWilcoxon-M ann-Withiney.

Tabela 2. Localização das úlceras por pressão por região do corpo.

\begin{tabular}{lcc}
\hline Localização da úlcera por pressão & $\begin{array}{c}\text { Aparecimento de novas úlceras } \\
\text { em pacientes com úlcera sacra }\end{array}$ & Prevalência \\
\hline Calcanho & $20 \%$ & - \\
Cotovelo & $6 \%$ & - \\
Escápula & $6 \%$ & - \\
Ísquio & $13 \%$ & - \\
oelho & $6 \%$ & - \\
Maléolo & $20 \%$ & - \\
Occipício & $6 \%$ & $94 \%$ \\
Sacra & - & - \\
Trocanter & $6 \%$ & - \\
\hline
\end{tabular}


Levantamento de custo médio de curativo industrializado por paciente por dia na Clínica Neurocirúrgica

Dentre os 62 pacientes entrevistados, 18 apre sentavam bai xos escores na Escala de Braden, ou seja, al to risco de desenvolver úlcera por pressão. A prevalência entre os pacientes da N eurocirurgia foi de $94 \%$ dentro da população desteestudo. A Tabela 3 mostra o custo médio diário para quatro pacientes que foram acompanhados desde que foram admitidos na Neurocirurgia. 0 custo diário aumentou à medida que aumentou o grau de destruição tecidual, pois foram utilizados, neste caso, curativos industrializados a fim defacilitar o debridamento, diminuir o odor das feridas e até mesmo como fator de prevenção para evitar queaúlcera grau I evoluísse para grau de maior dano tecidual. Nota-se também que todos esses pacientes apresentaram escores iguais ou menores que 16 (ponto crítico da escala).

H ouve dois casos (pacientes 1 e 2) em que os pacientes receberam assistência das famílias para prevenir tal comorbidade, fazendo mudança de decúbito a cada duas horas, higiene corporal adequada e massagem com ácidos graxos uma vez ao dia, cuidados com a alimentação eainda troca do colchão caixa de ovo por colchão d'água.

Foram também utilizados pela equipe de enfermagem curativos de poliuretano $5,1 \times 7,6 \mathrm{~cm}$, inicialmentea cada cinco dias e posteriormentea cada sete dias para o paciente 2 .

0 paciente 3 foi admitido na neurocirurgia com UP em grau II, necessitou fazer debridamento com hidrogel com alginato de cálcio, 0 que encareceu seu tratamento. 0 paciente 4 foi 0 mais crítico do grupo. Segundo dados do prontuário, o paciente foi admitido com UP grau I, mas evoluiu rapidamente para grau III enecessitou fazer debridamento com curativo de hidrogel e ainda carvão ativo, além da necessidade do uso de antimicrobiano e debridamento cirúrgico. Os pacientes que receberam algum tipo de prevenção apresentaram os menores custos em relação aos outros. 0 custo médio diário do paciente 4 superou o custo médio diário do paciente $1 \mathrm{em} 45 \%$.

No entanto, apenas quatro dos 18 pacientes que apresentaram UP usaram curativos industrializados. Seu uso foi verificado apenas em caso de grande gravidade para debridamento (hidrogel ecarvão ativo) ou em caso de prevenção (poliuretano).

Foi verificado que o uso de carvão ativo, hidrogel com alginato e alginato em placa aumentam o custo médio diário em $9 \%$, poisfoi utilizado apenas um de cada tipo para facilitar o debridamento cirúrgico, com isto seu custo fica diluído no custo médio diário, apesar de seu alto custo unitário.

Os curativos de poliuretano aumentam 0 custo médio diário do tratamento em $0,9 \%$, sendo usados semanalmente, mostrando-se mais acessíveis economicamente.

Os dados encontrados indicam que se houver medidas adequadas de prevenção com avaliação do paciente, usando escalas de estimativas de risco, a maioria dos casos de U P pode ser evitada. N este ponto ressalta-se a importância de serem institucionalizados protocolos de prevenção e tratamento, como os desenvolvidos nos Estados Unidos através da criação de um ato público queestabeleceu a Agency for $\mathrm{H}$ ealth Care Policy Research (AHCPR) ${ }^{19}$.

O Hospital da Restauração, como uma instituição pública de saúdeespecializada em politraumatismo, tem uma demanda superior a sua capacidade de atendimento, o que propicia uma situação crítica. M esmo quando os pacientes são recebidos na enfermaria, foi verificado que eles não recebem prevenção sistemática para UP durantesua permanência nesta instituição, enquan-

\begin{tabular}{|c|c|c|c|c|c|}
\hline Paciente & $\begin{array}{l}\text { Custo diário } \\
\text { médio }\end{array}$ & $\begin{array}{c}\text { Risco na } \\
\text { Escala de Braden }\end{array}$ & M edicamento & Curativo industrializado & $\begin{array}{l}\text { Grau de } \\
\text { UP }\end{array}$ \\
\hline 1 & $\mathrm{R} \$ 98,90$ & 13 & & & Grau 0 \\
\hline 2 & $R \$ 107,60$ & 13 & - & Poliuretanō 5,6x7,6cm & Grau I \\
\hline 3 & $\mathrm{R} \$ 122,00$ & 16 & & Poliuretano 5,6x7,6cm e & Grau II \\
\hline 4 & $\mathrm{R} \$ 180,00$ & 13 & $\begin{array}{c}\text { Sulbactam + } \\
\text { Ampicilina F/A } 3 G\end{array}$ & $\begin{array}{l}\text { Hidrogel c/alginato de cálcio } \\
\text { Carvão ativado } 10 \times 10 \mathrm{~cm} \text { e } \\
\text { hidrogel } 15 \times 20 \mathrm{~cm}\end{array}$ & Grau III \\
\hline
\end{tabular}


to os cuidados básicos de higiene são dispensados, na maioria das vezes, por seus familiares ou acompanhantes que geralmente não possuem conhecimento técnico para avaliar complicações dessa natureza. Esse fato reflete a dificuldade do serviço público dessa instituição, pois são insuficientes os profissionais de enfermagem por pacientes: geralmente um auxiliar para cada dez ou onze pacientes. Considerando-se que são pacientes graves quenecessitam de mudança de decúbito a cada duas horas e ainda cuidados gerais e de higiene, e que muitos deles são pacientes obesos ou com lesão cervical e que não devem ser movidos até avaliação médica adequada, deveria haver maior número de profissionais suficientes e devidamente treinados para lidar com esse tipo de paciente. Além disto, deve haver equipamentos adequados para prevenção das úlceras.

Foi apresentado um estudo em $1996^{21}$ no qual as autoras comparam o custo do tratamento de UP para pacientes quea desenvolveram após dois tipos de procedimentos (ponte de safena em artéria coronariana e reconstrução da bacia), durante os anos de 1990, 1991 e 1992. A média do tempo de estadia de todos os pacientes que realizaram esses procedimentos foi calculada em termos de custos para a instituição e comparada com aquela dos pacientes que fizeram os mesmos procedimentos, mas desenvolveram úlcera por pressão.

O estudo utilizou um questionário para obter informações além daquelas obtidas em prontuário médico. Segundo as autoras, o questionário abrangia relato do aparecimento da UP e documentos relativosà prevenção eao tratamento da úlcera. Foi comparada a incidência de UP a cada ano para cada um dos procedimentos descritos anteriormente. Os resultados foram mostrados por número de pacientes com UP para os dois procedimentos nos anos de 1990, 1991 e 1992. Para os pacientes que fizeram ponte de safena, foram $3,8 \%, 1,6 \%$ e $2,9 \%$, respectivamente, e para os pacientes que fizeram a reconstrução de bacia, foram $10,2 \%, 7,9 \%$ e 3,3\%, respectivamente. Segundo as autoras, a incidência diminuiu devido ao programa de prevenção desenvolvido e implementado durante o mesmo estudo. Não foram realizados estudos de prevalência. D os 2.239 pacientes que se submeteram ao procedimento de ponte de safena, 63 desenvolveram UP, e dos 823 pacientes que fizeram reconstrução da bacia, 56 desenvolveram a úlcera, durante os três anos de estudo.

Também foram calculados os custos adicionais de estadia no hospital para os pacientes que desenvolveram UP nos dois procedimentos, ponte de safena e reconstrução da bacia; no entanto, esses custos não consideram o gasto com os cuidados adicionais dispensados pela enfermagem em virtude do tratamento da úlcera. 0 custo adicional de ambos os procedimentos para os pacientes com UP foi deUS $\$ 237.587,70 \mathrm{em} 1990$, US\$ $151.613,70 \mathrm{em} 1991 \mathrm{edeUS} \$ 130.893,00 \mathrm{em}$ 1992. De acordo com as autoras, a diminuição da incidência de úlcera por pressão tornou possível a redução dos custos relativos aos adicionais de estadia.

É interessante notar que essas autoras usaram como base para o cálculo dos custos apenas os valores da estadia hospitalar, e segundo elas esses custos não correspondem a custos de cuidados da enfermagem ou produtos necessários para o tratamento.

Não há como confrontar diretamente os dados relativos a custos do estudo descrito anteriormente com a presente pesquisa, em razão de diferenças marcantes nos objetivos dos dois trabalhos e ainda no tempo de pesquisa de campo para obtenção dos dados. Os dados sobre incidência, por exemplo, não são comparáveis com este trabalho, pois os pacientes internados na N eurocirurgia em sua grande maioria já apresentavam pelo menos uma úlcera por pressão na sua admissão. Neste trabalho, as autoras não coletam dados relativos à prevalência. 0 s dados sobre custo podem ser considerados comparáveis em al guns aspectos, pois é certo que os custos aumentam à medida que aumentam os casos de UP, e que medidas de prevenção diminuem grandemente os custos das instituições de saúde.

Em 1989, foi desenvolvido um estudo comparativo para determinar o custo de produtos para prevenção de úlceras e o custo de produtos para tratamento ${ }^{22}$. Nesta pesquisa, o custo da hospitalização daqueles que desenvolveram úlcera ou dos que foram internados com úlcera foi comparado com o daqueles que não possuíam risco. A taxa de incidência foi de $1,5 \%$, ea taxa de prevalência foi de $14,5 \%$. 0 custo do tratamento foi de US\$11,95 ao dia em relação ao material, enquanto o custo da prevenção foi de US $\$ 4,83$.

Nesse estudo, os autores chegam à mesma conclusão que o presente estudo: a prevenção de UP émenos dispendiosa que o tratamento. Quando comparamos a taxa de prevalência obtida pela autora (14,5\%) com a obtida no presente estudo $(22,6 \%)$ verifica-sequeesta é superior àquela em $36 \%$. 0 custo diário do tratamento calculado por esses autores considerou apenas os produtos usados pelo grupo de tratamento e da preven- 
ção, enquanto neste estudo foi feita uma avaliação mais global, considerando diária hospitalar, materiais médico-hospitalares, custos com mé dicos e enfermagem e medicamentos.

Em 1990, foi feito um estudo do custo do tratamento deU $P$ em 27 pacientes em um hospital do Distrito de Saúde do Reino Unido, que durou um mês ${ }^{23}$. No cálculo do custo direto incluíram-se os valores de produtos farmacêuticos, material para curativos e o tempo gasto pelo enfermeiro. 0 valor mensal do tratamento foi de 1.618,00 libras, e a estimativa anual seria de 19.416,00 libras.

Esse trabalho assemelha-se com o presente em alguns aspectos, pois já inclui no cálculo dos custos do tratamento de UP o gasto com o profissional de enfermagem; no entanto, é menos abrangente, pois não inclui os custos da diária hospitalar e ainda não faz a comparação com o custo relativo à prevenção. É bastantedifícil comparar os dados obtidos nos estudos realizados nessa área, pois geralmente não foram realizados dentro de parâmetros padronizados, ou seja, não consideram para efeito de custo os mesmos componentes, eainda pelo fato deocorrerem dificuldades nos cál culos relativos à prevalência e incidência, porqueàs vezes tais conceitosnão são claramenteidentificados.

\section{Conclusão}

Este estudo realizado na Clínica N eurocirúrgica usou uma pequena amostra do grande número de pacientes que são atendidos por esta instituição, no entanto consegue fazer um retrato da realidade encarada por ela, que se imagina não ser muito diferente de outras instituições públicas encontradas por todo o país.

H oje, a úlcera por pressão éum problema de saúde pública, por isso muitos esforços têm sido feitos para melhorar os cuidados com os pacientes, e atualmente úlcera por pressão é utilizada como indicador de qualidade dos serviços de saú$\mathrm{de}^{17}$. No entanto, não se pode imaginar que seja dever apenas das equipes de enfermagem a solução dessa problemática, mas sugerimos que devam ser formadas equipes multiprofissionais dentro dos hospitais, trabalhando em conjunto, buscando soluções baseadas em trabalhos científicos, trazendo as descobertas científicas para a realidade e para a prática diária da instituição. Deve-se fazer treinamentos com a equipe de enfermagem para que sejam identificados os indivíduos em risco de desenvolver úlcera por pressão usando um protocolo de medidas preventivas apresentado durante treinamento e ainda identificar os fatores críticos de desenvolvimento de úlcera por pressão.

De acordo com os dados coletados neste estudo, conclui-sequeémuito mais vantajoso prevenir a úlcera por pressão do que permitir que ela se instale para depois tentar tratá-la, pois o desgaste é muito maior para as equipes de enfermagem e para a equipe médica, e o custo é muito maior para o sistema de saúde. Para o paciente, o desgaste é imensurável, e o trauma é uma ferida na alma que marca para o resto da vida.

\section{Colaboradores}

ACB Lima foi a responsável pela concepção, pelo delineamento, análise, interpretação, revisão e aprovação da versão publicada; DM Guerra, pela revisão crítica, orientação da redação e aprovação da versão publicada. 


\section{Referências}

1. Caulliraux HM, Yuki M. Gestão pública e reforma administrativa: conceitos e casos: a experiência de Florianópolis. Rio de Janeiro: Lucerna; 2004.

2. Bueno VJM. Valorización del riesco de aparición de ulceras de presión en la unidad de cuidados intensivos. Rev Eletrônica de M edicina Intensiva 2005; 5(11):art.923 [periódico na Internet] [acessado 2006 jul 31]. Disponível em: http://remi.uninet.edu/ 2005/11/REMI/0923.htm

3. Gosnell DJ. An assessment tool to pressure sores. N urs Res 1973; 22(1):55-59.

4. Waterlow J. Recording risk. Nurs Times 1995; 91(11):64-66.

5. M arcer L. Pressure area care: monitoring standards. Nurs Stand 1992; 12(47):4-7.

6. Braden BJ, Bergstrom N. Clinical utility of Braden Scale for predicting pressure sores risk. Decubitus 1989; 2(3):44-51.

7. Allman RM. Epidemiology of pressure sores in different populations. Decubitus 1989; 2(2):30-33.

8. Panel for prediction and prevention of pressure ulcer in adults. Rockville: Agency for Health Care Policy and Research, Public Health Service, Department of Health and Human Services: US; 1992.

9. Hanson DS, Langemo D, Olson B, Hunter S, Burd C. Evaluation of pressure ulcer prevalence rates for hospice patients post-implementation of pressure ulcer protocols. The Am J Hospice \& Paliative Care 1994; 11(6):14-19.

10. Knox DO. Efects of different turn intervals on skin of helth older adults. Advancesing Wound Care 1994; $7(1): 48-56$

11. Pernerger TV, Héliot C, Raë AC, Gaspoz JM. Hospital acquire pressure ulcers: risc factors and use of preventive devices. Arch Interm M ed 1998; 158:19401945.

12. Ooi LW, Morris JN, Brandeis HG, Hossain M, Lipsitz LA. Nursing home characteristics and the development of pressure sores and disruptive behaviour. British Geriatrics Society Age and Ageing 1999; 28:45-52.

13. Van Marum RJ, Ooms ME, Ribbe MW, Van Eijk JT. The Dutch pressure sore assessment score or Norton Scale for identifying at-risk nursing home patients? British Geriatrics Society Age and Ageing 2000; 29:63-38.

14. Courtenay M, Church JCT, Ryan TJ. Larva therapy in wound management. J R Soc M ed 2000; 93:72-74.
15. Royal College of Nursing. Clinical Pratice Guidelines. Pressure ulcer risk assessment and prevention. 2001. London: RCN; 2001.

16. Porras IG, Rosillo CS. Úlceras por presión: nueva escala de valoración para pacientes críticos. Rev Eletrônica de M edicina Intensiva 2001; 1(6):art. 148. [periódico na Internet] [acessado 2006 jul 31]. Disponível em: http://www.remi.uninet.edu/2001/06/ REM /0148.htm

17. Bueno VJM. Efecto beneficioso de los ácidos hiperoxigenados en la prevención de las úlceras por présion. Rev Eletrônica de M edicina Intensiva 2003; 3(1):art.538. [periódico na Internet] [acessado 2006 jul 31]. Disponível em: http://remi.uninet.edu/ 2005/11/REMI/0528.htm

18. Uhlemann C, Heinig B, Wollina U. Therapeutic ultrasound in lower extremity wound management. Lower Extremity Wounds 2003; 2(3):152-157.

19. Jorge AS, Dantas SR. Abordagem multiprofissional do tratamento de feridas. São Paulo: Ateneu; 2003.

20. Blanes L, Duarte I, Calil JA, Ferreira LM . Avaliação clínica e epidemiológica das úlceras por pressão em pacientes internados no Hospital São Paulo. Revista da Associação M édica Brasileira 2004; 50(2):182-187.

21. Lapsley HM, Vogels R. Cost and prevention of pressure ulcer in an acute teaching hospital. International Journal for Quality in Health Care 1996; 8(1):6166.

22. Oot-Giromini B, Bidwell FC, Heller NB, Parks $M L$, Prebish Em, Wicks P, Williams PM. Pressure ulcer prevention versus treatment: comparative product cost study. Decubitus 1989; 2(3):52-54.

23. Hale C. Pressure sores: assessing the cost. Nursing Times 1990; 86(25):66-69.

Artigo apresentado em 28/08/2007

Aprovado em 30/11/2007

Versão final apresentada em 30/12/2007 